\title{
Fire Intensity Effects on the Understory in Ponderosa Pine Forests
}

\author{
CHARLES D. ARMOUR, STEPHEN C. BUNTING, LEON F. NEUENSCHWANDER
}

\begin{abstract}
The effect of fire intensity on understory vegetation on seral stands of ponderosa pine are presented. Vegetational recovery for 2 burn intensities and unburned sites were compared. The results suggest a change in the understory dominance structure following underburning is related to the amount of duff consumed by the fire and independent of fire line intensity and flame length. A significantly greater proportion of duff was removed on high fire intensity sites $(80 \%)$ than on low intensity sites $(40 \%)$. However, the variation in fire line intensity was great, with some overlap. Fire line intensity ranged from 30 to $3,034 \mathrm{kcal} / \mathrm{m}-\mathrm{s}$ on high intensity sites and from 25 to $194 \mathrm{kcal} / \mathrm{m}-\mathrm{s}$ on low intensity sites. Flame length ranged from 0.1 to $1.7 \mathrm{~m}$ on both high and low intensity sites. Graminoid canopy coverage was lowest on high intensity sites. The reduction was apparently the result of prolonged smoldering of the duff layer. There was no significant difference in coverage among treatments for shrubs or forbs. A significant change in frequency among treatments was noted for 11 of 54 species sampled.
\end{abstract}

Prescribed burning can be an effective tool in managing seral stands of ponderosa pine (Pinus ponderosa). Fire may be used to reduce fuel loading, thin thickets of saplings, create mineral seedbed for seed germination, remove shade tolerant trees from the understory, prune the lower branches from trees, and reduce needle litter on the soil surface (Biswell et al. 1973). Prescribed burning can also be an effective tool for understory vegetation management. Herbaceous yields are dependent on the percent tree overstory canopy closure and the amount of accumulated litter (Wright 1978). Different intensity fires may be used to manipulate the total tree canopy and litter depth. The effect fire intensity may have on understory development has not been previously studied. The objective of this study was to evaluate the effect of fire intensity on early vegetational development in ponderosa pine communities of the Douglas-fir/ninebark (Pseudotsuga menziesii/ Physocarpus malvaceus) habitat type (Daubenmire and Daubenmire 1968).

In the northern Rocky Mountains, vegetational recovery in response to fire that removes the overstory is variable, although the eventual outcome is relatively consistent (Lyon and Stickney 1976, Cattelino et al. 1979). Early successional seres apparently lack the consistency and predictability exhibited by older seres (Cattelino et al. 1979). In general, however, succession proceeds in a more or less orderly sequence from herb dominated communities, to shrub dominated communities, and finally, tree dominated communities (Larsen 1929, Lyon and Stickney 1976). The number of years the

\footnotetext{
Authors are graduate assistant, assistant professor of range resources, and associate professor of forest resources, College of Forestry, Wildlife, and Range Sciences, University of Idaho, Moscow.

Research was supported by the USDA Forest Serv., Intermt. Forest and Range Experiment Station, N. Forest Fire Laboratory, through cooperative agreement 12-11-204-11 and the Forest, Wildlife and Range Exp. Sta., Univ. of Idaho Contribution No. 245.

Manuscript received November 5, 1982.
}

first 2 phases dominate the site is dependent on the severity of the disturbance (Zamora 1982) and other site characteristics.

In Douglas-fir habitat types, fire promotes the establishment and growth of ponderosa pine (Wright and Bailey 1982). Seral stands of ponderosa pine reproduce best in clearings (Schubert 1974) with a mineral soil surface (Schultz and Biswell 1959). In the absence of fire, Douglas-fir may supplant ponderosa pine and become the overstory dominant (Daubenmire and Daubenmire 1968). Ponderosa pine is very fire resistant because of its thick bark, deep root system, and moderately high, open crown (Flint 1925).

Many shrubs regenerate above-ground tissue from underground organs following fire in the Douglas-fir/ninebark habitat type. Sprouting from root crowns and rhizomes are 2 adaptations that enable shrubs to survive fires. Over $50 \%$ of all shrub species in the northern Rocky Mountains regenerate from root crowns. Some shrubs also recolonize burned sites with dormant, fire-activated seeds (Leege 1968, Lyon and Stickney 1976).

Several studies have documented shrub response to fire. In northern Idaho, shrubs resprout well after spring and fall fires. Fall fires stimulate greater resprouting than spring fires, however (Hooker 1972). A recent northern Idaho study showed shrubs dominate the grand fir/myrtle pachistima (Abies grandis/Pachistima myrinites) habitat type (Daubenmire and Daubenmire 1968) from about 8 to 27 years after burning (Zamora 1982). In southern Idaho, shrub cover was 10 times the preburn level 7 years after burning (Lyon 1971).

The most important fire survival mechanism for on-site herbaceous plants is the rhizome; off-site species recolonize burned areas by wind-borne seed (Lyon and Stickney 1976). In southern Idaho, Lyon (1971) found herbaceous cover was nearly as dense I year after burning as in unburned areas. In the grand fir/myrtle pachistima habitat type, herbaceous species dominated the site from the first through the eighth year after burning (Zamora 1982). Keown (1978) summarized the fire adaptations and post-fire responses for specific herbaceous and shrub species.

\section{Methods}

This study was conducted on the Coeur d'Alene Indian Reservation in Benewah County, Ida. Elevation of the study area is approximately $880 \mathrm{~m}$ above sea level on gently rolling hills. Average annual precipitation is $560 \mathrm{~mm}$, the majority of which falls from October to March. The study area has been previously described by Bakken (1981). A 10-cm layer of volcanic ash from the May 18, 1980, eruption of Mt. St. Helens was deposited on the area.

Sampled sites were limited to the Douglas-fir/ninebark habitat type. The sites were dominated by seral stands of ponderosa pine 
with an understory of shrubs and perennial graminoids. The study areas were selectively logged 3 times at about 10 -year intervals, the last time in 1977 . Between 11 and $35 \%$ of the overstory basal area was removed from all of the sites. The amount of basal area removed on each site was proportional to the amount removed from the entire stand. Before forest management activities, including fire suppression, the sites had a mean fire-free interval of approximately 22 years (unpublished data). However, the sites have not burned for about 44 to 62 years. Grazing by domestic livestock has not occurred on the sampled sites for at least 30 years.

A split-block design was used to test the null hypothesis that there is no difference in understory species cover or frequency among treatments. Three treatments were identified: unburned control, low intensity, and high intensity burns. Three areas of similar stand history and composition were located within $25 \mathrm{~km}$ of each other. Each location was subdivided into nine 0.2 to 1.0 ha units and burned under different intensities during the fall of 1978 . The 6 burned units at each location were treated during a 30-day period under varying temperature, fuel moisture and relative humidity conditions. This resulted in fires of varying intensity. Three replications of high and low intensities and unburned sites were sampled within each location. There was a large degree of variation in fire line intensity $(C . V .=143.1)$ between fire treated sites, with some overlap in intensity. Fire line intensity (Van Wagner 1973) was significant $(P<0.05)$, and ranged from 30 to $3,034 \mathrm{kcal} / \mathrm{m}$-s on high intensity sites and from 25 to $194 \mathrm{kcal} / \mathrm{m}-\mathrm{s}$ on low intensity sites. The product of rate-of-spread, the amount of fuel consumed, and the heat content of wood $(450 \mathrm{cal} / \mathrm{gm})$ yields fire line intensity. Flame length averaged $0.9 \mathrm{~m}$, with a range of 0.1 to $1.7 \mathrm{~m}$. Percent area burned and duff reduction were measured the first season after treatment.

Vegetational development was measured for 3 consecutive years after treatment by monitoring permanent plots. Five $10-\mathrm{m}$ transects were located in each $15 \times 15 \mathrm{~m}$ macroplot. Shrub canopy coverage was measured by the line intercept method (Canfield 1941). Herbaceous canopy coverage and frequency were measured by permanently locating ten $20 \times 50 \mathrm{~cm}$ microplots along each of the 5 shrub transects. Cover was estimated using canopy coverage classes (Daubenmire 1959). Frequency for shrub and tree seedlings was determined with the microplots. Duff depth was measured at one point in each microplot. Taxonomy of plant species follows Hitchcock and Cronquist (1973).

Jaccard's Index of Similarity (Mueller-Dombois and Ellenberg 1974) was used to test for understory community differences among locations and treatments. Canopy coverage and frequency differences were analyzed with the Statistical Analysis System's (Helwig and Council 1979) ANOVA procedure. Duncan's multiple range test ranked statistically significant means. The level of significance was set at $P<0.10$.

\section{Results and Discussion}

The 3 locations studied were very similar in species composition and abundance prior to the burn. The rich array of species was the result of the disturbance by logging and fire and because the sampled sites were on the mesic end of the habitat type. Five shrubs, 4 perennial graminoids, 8 perennial forbs, and 4 annuals displayed significant differences in canopy coverage among treatments. Other species had canopy coverage values less than 5\%; therefore, successional trends of individual species could not be assessed.

Canopy coverage for graminoids was significantly lower on high intensity sites during all years after treatment (Fig. 1). Cover decreased from $20.4 \%$ on unburned sites to $10.9 \%$ on high intensity sites. Conversely, there was no significant difference in canopy
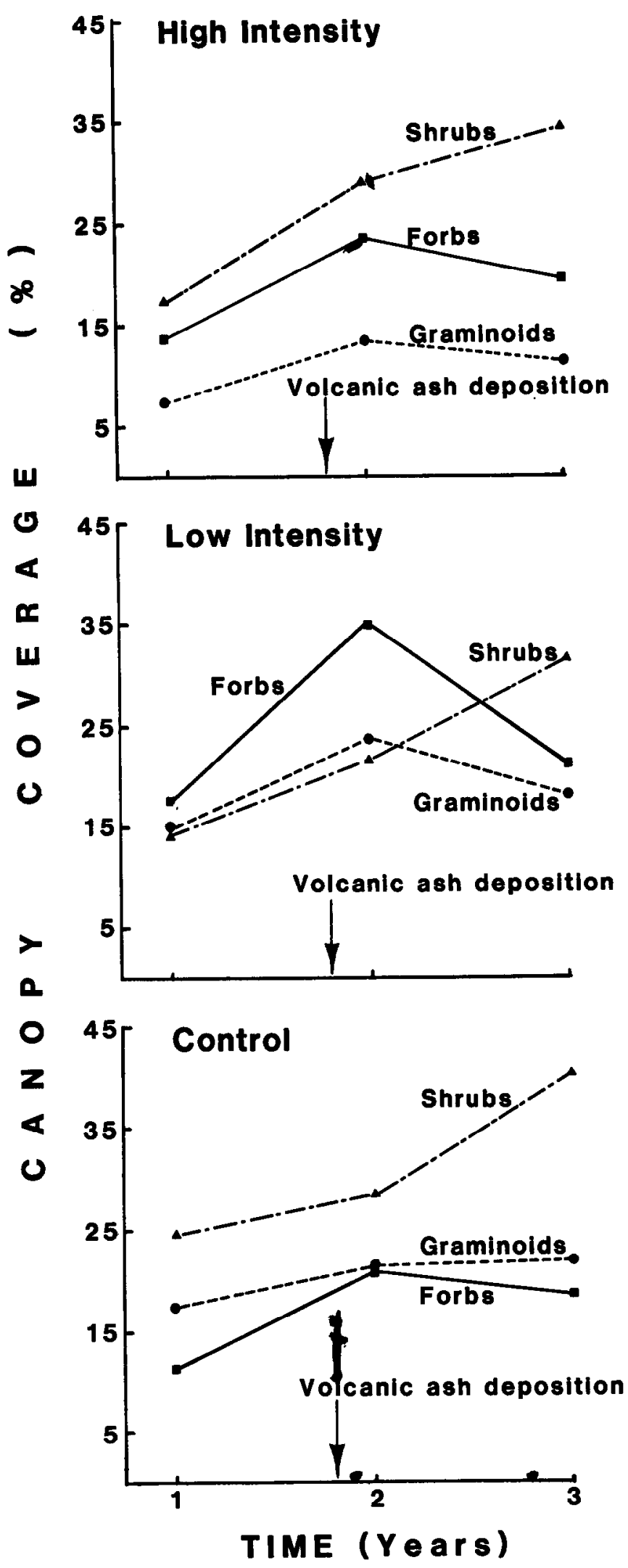

Fig. 1. Mean canopy coverage of understory vegetation on unburned sites and sites treated with low and high intensity fires in relation to the number of years after treatment. 
coverage for shrubs or forbs among treatments. Shrub coverage was $27 \%$ on high intensity sites, $23 \%$ on low intensity sites, and $31 \%$ on unburned sites. Forb coverage averaged 20, 25, and 17\%, respectively, on similarly treated sites.

Canopy coverage for graminoids significantly increased from the first through the third year after treatment as compared to the unburned sites (Fig. 1). A large increase in coverage was observed between the first and second year. Likewise, the increased canopy coverage for forbs was significantly greater than the unburned sites for all 3 years and followed the same pattern displayed by graminoids. Canopy coverage for shrubs significantly increased over the 3-year observation period.

A significant change in frequency among treatments was noted for 11 of 54 species sampled (Fig. 2). Columbia brome (Bromus vulgaris) was the only common species harmed by fire. It was 2 to 5 times more frequent on unburned sites than on high intensity sites. The difference between unburned sites and low intensity sites was much less. The other 10 species were not always harmed by fire. Creeping Oregongrape (Berberis repens), field woodrush (Luzula campestris), and stra wberry (Fragaria virginiana) were harmed on high intensity sites, but not on low intensity sites. Creeping Oregongrape was nearly absent from high intensity sites. Field woodrush was more than 7 times more frequent on unburned sites than on high intensity sites. Strawberry was more than twice as common on unburned sites and low intensity than on high intensity sites.

Conifer seedlings were nearly absent from all sampled sites during all 3 years. Redstem ceanothus (Ceanothus sanguineus) seedlings were found only on high intensity sites. Similarly, spreading dogbane (Apocynum androsaemifolium) was rare on sites other than high intensity sites. Fireweed (Epilobium angustifolium) was 2 to 5 times more common on high intensity sites than low intensity sites and was extremely rare on unburned sites. Narrowleaf collomia (Collomia linearis) was commonly found on treated and untreated plots, but it was most common on high intensity sites. Frequency of collomia for unburned sites and low intensity and high intensity sites ranged from 1 to 10,9 to 14 , and 10 to $37 \%$, respectively.

Slender cinquefoil (Potentilla gracilis), prairie smoke avens (Geum triflorum), and American vetch (Vicia americana) were more common on low intensity sites than on the other 2 sites. Mean frequency for slender cinquefoil ranged from 26 to $32 \%$ on low intensity sites, 7 to $11 \%$ on high intensity sites, and 18 to $22 \%$ on unburned sites. Frequency for prairie smoke avens and American vetch was from 2 to 4 and 7 to 11 times greater on low intensity sites than on unburned sites or high intensity sites.

There was a significant difference in frequency from the first through the third growing season for 9 of the above 11 species. Only redstem ceanothus seedlings exhibited a decrease in frequency during the course of the study. Field woodrush, strawberry, prairie smoke avens, slender cinquefoil, and narrow-leaf collomia increased over the observation period. The increase in frequency for Columbia brome and American vetch was followed by a decline the third year after the fire.

The reduction in canopy coverage for graminoids on high intensity sites was apparently the result of prolonged duff smoldering, which destroyed root crowns and rhizomes. A significantly greater proportion of duff was removed on high intensity sites. Duff depth of high intensity sites was $1.3 \mathrm{~cm}, 4.0 \mathrm{~cm}$ on low intensity sites, and $6.6 \mathrm{~cm}$ on unburned sites. Duff depth means

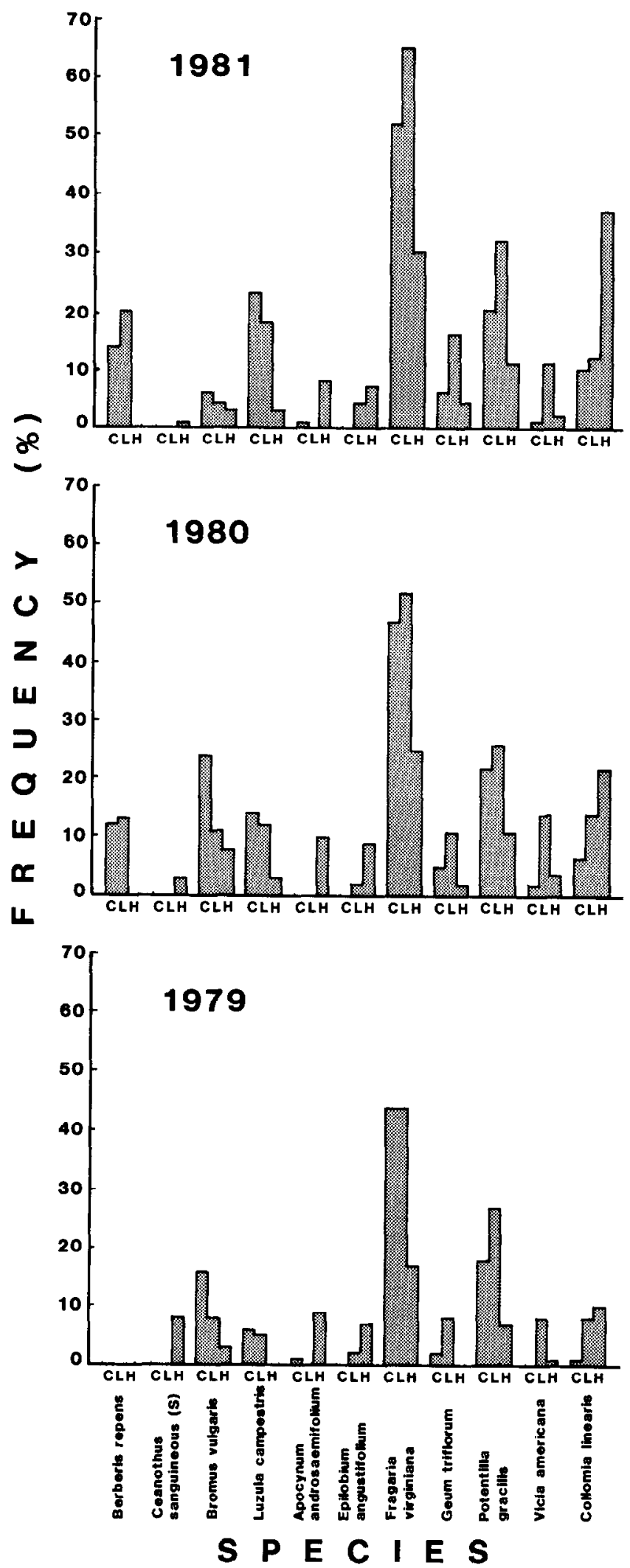

Fig. 2. Mean frequency of understory species which changed significantly on unburned sits, and sites treated with low and high intensity fires in relation to the number of years after treatment $(C=$ unburned, $L=$ low intensity, $H=$ high intensity). 
Table 1. Mean canopy coverage and frequency ${ }^{1}$ of shrub and perennial graminoid species on unburned and high and low intensity prescribed burned sites in a ponderosa pine community.

\begin{tabular}{|c|c|c|c|c|c|c|c|c|c|c|c|c|}
\hline \multirow[b]{2}{*}{ Species } & \multicolumn{6}{|c|}{ Treatment (ave. across years) } & \multicolumn{6}{|c|}{ Time since treatment (yrs) (ave. across treatments) } \\
\hline & Cont & & Low & & Hig & & 1 & & 2 & & 3 & \\
\hline $\begin{array}{l}\text { Amelanchier alnifolia } \\
\text { Berberis repens } \\
\text { Ceanothus sanguineus } \\
\text { Crataegus douglasii } \\
\text { Holodiscus discolor } \\
\text { Physocarpus malvaceus } \\
\text { Prunus emarginata } \\
\text { P. virginiana } \\
\text { Rosa gymnocarpa } \\
\text { R. nutkana } \\
\text { R. woodsii } \\
\text { Salix scouleriana } \\
\text { Spiraea betulifolia } \\
\text { Symphoricarpos albus } \\
\text { S. mollis }\end{array}$ & $\begin{array}{r}0.5 \mathrm{a}^{2} \\
1.1 \mathrm{a} \\
0.2 \mathrm{a} \\
1.0 \mathrm{a} \\
3.9 \mathrm{a} \\
2.0 \mathrm{a} \\
0.0 \mathrm{a} \\
3.7 \mathrm{a} \\
3.1 \mathrm{a} \\
0.5 \mathrm{a} \\
0.8 \mathrm{a} \\
0.1 \mathrm{~b} \\
0.6 \mathrm{a} \\
17.3 \mathrm{a} \\
0.0 \mathrm{a}\end{array}$ & $8.6 \mathrm{ab}$ & $\begin{array}{r}0.2 \mathrm{a} \\
1.2 \mathrm{a} \\
0.1 \mathrm{a} \\
0.0 \mathrm{a} \\
1.0 \mathrm{a} \\
0.0 \mathrm{~b} \\
0.0 \mathrm{a} \\
5.0 \mathrm{a} \\
0.6 \mathrm{~b} \\
0.1 \mathrm{a} \\
0.7 \mathrm{a} \\
1.1 \mathrm{a} \\
1.8 \mathrm{a} \\
14.3 \mathrm{a} \\
0.0 \mathrm{a}\end{array}$ & $11,1 \mathrm{a}$ & $\begin{array}{r}0.1 \mathrm{a} \\
0.0 \mathrm{~b} \\
0.8 \mathrm{a} \\
0.2 \mathrm{a} \\
2.7 \mathrm{a} \\
0.4 \mathrm{~b} \\
0.1 \mathrm{a} \\
2.1 \mathrm{a} \\
0.9 \mathrm{~b} \\
0.2 \mathrm{a} \\
0.2 \mathrm{~b} \\
0.0 \mathrm{~b} \\
2.1 \mathrm{a} \\
20.4 \mathrm{a} \\
1.0 \mathrm{a}\end{array}$ & $0.2 \mathrm{~b}$ & $\begin{array}{r}0.1 \mathrm{~b} \\
0.0 \mathrm{~b} \\
0.1 \mathrm{~b} \\
0.4 \mathrm{a} \\
1.7 \mathrm{~b} \\
0.5 \mathrm{a} \\
0.0 \mathrm{a} \\
3.3 \mathrm{a} \\
1.2 \mathrm{~b} \\
0.0 \mathrm{~b} \\
0.6 \mathrm{a} \\
0.2 \mathrm{a} \\
1.4 \mathrm{a} \\
11.4 \mathrm{c} \\
0.0 \mathrm{a}\end{array}$ & $0.0 b$ & $\begin{array}{r}0.2 \mathrm{~b} \\
1.1 \mathrm{a} \\
0.2 \mathrm{~b} \\
0.2 \mathrm{a} \\
1.9 \mathrm{~b} \\
0.9 \mathrm{a} \\
0.0 \mathrm{a} \\
3.6 \mathrm{a} \\
1.1 \mathrm{~b} \\
0.1 \mathrm{~b} \\
0.8 \mathrm{a} \\
0.5 \mathrm{a} \\
2.0 \mathrm{a} \\
18.2 \mathrm{~b} \\
0.9 \mathrm{a}\end{array}$ & $8.4 \mathrm{a}$ & $\begin{array}{r}0.4 \mathrm{a} \\
1.3 \mathrm{a} \\
0.9 \mathrm{~b} \\
0.8 \mathrm{a} \\
3.9 \mathrm{a} \\
1.0 \mathrm{a} \\
0.1 \mathrm{a} \\
3.9 \mathrm{a} \\
2.2 \mathrm{a} \\
0.7 \mathrm{a} \\
0.4 \mathrm{a} \\
0.2 \mathrm{a} \\
1.0 \mathrm{a} \\
22.5 \mathrm{a} \\
0.0 \mathrm{a}\end{array}$ & $11.4 \mathrm{a}$ \\
\hline Perennial graminoids & & & & & & & & & & & & \\
\hline $\begin{array}{l}\text { Agropyron spicatum } \\
\text { Bromus vulgaris } \\
\text { Calamagrostis rubescens } \\
\text { Elymus glaucus } \\
\text { Festuca idahoensis } \\
\text { F. scabrella } \\
\text { Luzula campestris } \\
\text { Poa spp. } \\
\text { Trisetum canescens }\end{array}$ & $\begin{array}{r}1.4 \mathrm{a} \\
0.7 \mathrm{a} \\
29.1 \mathrm{a} \\
2.7 \mathrm{a} \\
1.9 \mathrm{a} \\
0.0 \mathrm{~b} \\
0.5 \mathrm{a} \\
2.8 \mathrm{a} \\
0.8 \mathrm{c}\end{array}$ & $\begin{array}{r}25.7 \mathrm{a} \\
15.5 \mathrm{a} \\
44.0 \mathrm{a} \\
46.1 \mathrm{a} \\
24.8 \mathrm{a} \\
0.6 \mathrm{a} \\
14.5 \mathrm{a} \\
29.0 \mathrm{a} \\
21.2 \mathrm{a}\end{array}$ & $\begin{array}{r}2.1 \mathrm{a} \\
0.3 \mathrm{~b} \\
27.8 \mathrm{a} \\
1.7 \mathrm{a} \\
1.0 \mathrm{a} \\
0.6 \mathrm{a} \\
0.4 \mathrm{a} \\
2.1 \mathrm{a} \\
0.5 \mathrm{~b}\end{array}$ & $\begin{array}{r}30.4 \mathrm{a} \\
7.7 \mathrm{~b} \\
48.1 \mathrm{a} \\
36.4 \mathrm{a} \\
20.4 \mathrm{a} \\
5.3 \mathrm{a} \\
11.6 \mathrm{a} \\
29.3 \mathrm{a} \\
15.6 \mathrm{~b}\end{array}$ & $\begin{array}{r}0.8 \mathrm{a} \\
0.3 \mathrm{~b} \\
16.1 \mathrm{a} \\
1.2 \mathrm{a} \\
0.4 \mathrm{a} \\
0.2 \mathrm{ab} \\
0.1 \mathrm{~b} \\
1.8 \mathrm{a} \\
0.2 \mathrm{c}\end{array}$ & $\begin{array}{r}11.8 \mathrm{a} \\
4.6 \mathrm{~b} \\
25.7 \mathrm{a} \\
24.4 \mathrm{a} \\
10.8 \mathrm{a} \\
2.1 \mathrm{a} \\
2.0 \mathrm{~b} \\
22.2 \mathrm{a} \\
5.7 \mathrm{c}\end{array}$ & $\begin{array}{r}1.3 \mathrm{~b} \\
0.5 \mathrm{a} \\
22.4 \mathrm{a} \\
1.5 \mathrm{~b} \\
0.6 \mathrm{c} \\
0.3 \mathrm{~b} \\
01 . \mathrm{b} \\
1.0 \mathrm{c} \\
0.1 \mathrm{c}\end{array}$ & $\begin{array}{r}22.7 \mathrm{a} \\
9.1 \mathrm{~b} \\
40.5 \mathrm{a} \\
27.3 \mathrm{c} \\
11.7 \mathrm{c} \\
2.4 \mathrm{ab} \\
3.9 \mathrm{c} \\
18.4 \mathrm{~b} \\
3.9 \mathrm{~b}\end{array}$ & $\begin{array}{r}1.7 \mathrm{a} \\
0.7 \mathrm{a} \\
26.2 \mathrm{a} \\
2.1 \mathrm{a} \\
1.0 \mathrm{~b} \\
0.1 \mathrm{c} \\
0.4 \mathrm{a} \\
3.3 \mathrm{a} \\
0.4 \mathrm{~b}\end{array}$ & $\begin{array}{r}23.6 \mathrm{a} \\
14.5 \mathrm{a} \\
41.6 \mathrm{a} \\
34.1 \mathrm{~b} \\
16.1 \mathrm{~b} \\
1.2 \mathrm{~b} \\
9.6 \mathrm{~b} \\
30.4 \mathrm{a} \\
8.7 \mathrm{~b}\end{array}$ & $\begin{array}{r}1.3 \mathrm{~b} \\
0.2 \mathrm{~b} \\
24.6 \mathrm{a} \\
2.0 \mathrm{a} \\
1.6 \mathrm{a} \\
0.4 \mathrm{a} \\
0.4 \mathrm{a} \\
2.2 \mathrm{~b} \\
0.9 \mathrm{a}\end{array}$ & $\begin{array}{r}21.5 \mathrm{a} \\
4.2 \mathrm{c} \\
35.7 \mathrm{~b} \\
45.5 \mathrm{a} \\
28.3 \mathrm{a} \\
4.3 \mathrm{a} \\
14.7 \mathrm{a} \\
31.8 \mathrm{a} \\
30.0 \mathrm{a}\end{array}$ \\
\hline
\end{tabular}

Numbers to the left is percent coverage; the number to the right is percent frequency.

2 Means within a row followed by same letters are not significantly different $(P<0.1)$.

Table 2. Mean canopy coverage and frequency' of perennial forbs and annuals on unburned and high and low intensity prescribed burned sites in a ponderosa pine community.

\begin{tabular}{|c|c|c|c|c|c|c|c|c|c|c|c|c|}
\hline \multirow{2}{*}{$\frac{\text { Species }}{\text { Perennial forbs }}$} & \multicolumn{6}{|c|}{ Treatment (ave. across years) } & \multicolumn{6}{|c|}{ Time since treatment (yrs) (ave. across treatments) } \\
\hline & & ntrol & & Low & & High & & 1 & & 2 & & 3 \\
\hline $\begin{array}{l}\text { Perennial forbs } \\
\text { Achillea millefolium } \\
\text { Anemoni piperi } \\
\text { Apocynum androsaemifolium } \\
\text { Arenaria macrophylla } \\
\text { Aster jessicae } \\
\text { Cirsium spp. } \\
\text { Epilobium angustifolium } \\
\text { Fragaria virginiana } \\
\text { Galium boreale } \\
\text { Geranium viscosissimum } \\
\text { Geum triflorum } \\
\text { Hieracium albiflorum } \\
\text { Lathyrus bijugatus } \\
\text { Lomatium triternatum } \\
\text { Lupinus spp. } \\
\text { Osmorhiza chilensis } \\
\text { Penstemon spp. } \\
\text { Potentilla glandulosa } \\
\text { P. gracilis } \\
\text { Pteridium aquilinum } \\
\text { Solidago canadensis } \\
\text { Stellaria spp. } \\
\text { Trifolium latifolium } \\
\text { Vicia americana }\end{array}$ & $\begin{array}{l}1.0 \mathrm{a}^{2} \\
0.0 \mathrm{a} \\
0.0 \mathrm{~b} \\
1.3 \mathrm{a} \\
0.3 \mathrm{~b} \\
0.1 \mathrm{~b} \\
0.0 \mathrm{~b} \\
2.9 \mathrm{ab} \\
2.7 \mathrm{a} \\
0.9 \mathrm{a} \\
0.3 \mathrm{~b} \\
0.7 \mathrm{a} \\
0.7 \mathrm{a} \\
0.4 \mathrm{a} \\
0.1 \mathrm{a} \\
0.1 \mathrm{a} \\
0.5 \mathrm{a} \\
0.4 \mathrm{a} \\
1.4 \mathrm{ab} \\
1.3 \mathrm{a} \\
0.5 \mathrm{a} \\
0.6 \mathrm{a} \\
1.9 \mathrm{a} \\
0.1 \mathrm{~b}\end{array}$ & $\begin{array}{r}28.2 \mathrm{a} \\
1.9 \mathrm{a} \\
0.7 \mathrm{~b} \\
11.0 \mathrm{a} \\
3.9 \mathrm{~b} \\
1.0 \mathrm{~b} \\
0.2 \mathrm{~b} \\
47.6 \mathrm{a} \\
24.4 \mathrm{a} \\
12.7 \mathrm{a} \\
4.2 \mathrm{~b} \\
2.7 \mathrm{a} \\
13.9 \mathrm{a} \\
4.5 \mathrm{a} \\
1.4 \mathrm{a} \\
2.7 \mathrm{a} \\
6.2 \mathrm{a} \\
5.6 \mathrm{a} \\
20.2 \mathrm{c} \\
6.5 \mathrm{a} \\
3.2 \mathrm{a} \\
4.4 \mathrm{a} \\
25.5 \mathrm{a} \\
1.2 \mathrm{~b}\end{array}$ & $\begin{array}{c}1.3 \mathrm{a} \\
0.1 \mathrm{a} \\
0.0 \mathrm{~b} \\
2.1 \mathrm{a} \\
2.2 \mathrm{a} \\
0.1 \mathrm{~b} \\
0.3 \mathrm{~b} \\
3.4 \mathrm{a} \\
3.5 \mathrm{a} \\
1.7 \mathrm{a} \\
0.8 \mathrm{a} \\
0.8 \mathrm{a} \\
0.4 \mathrm{a} \\
0.4 \mathrm{a} \\
0.6 \mathrm{a} \\
0.2 \mathrm{a} \\
0.2 \mathrm{a} \\
0.4 \mathrm{a} \\
2.2 \mathrm{a} \\
2.3 \mathrm{a} \\
1.1 \mathrm{a} \\
0.6 \mathrm{a} \\
1.2 \mathrm{a} \\
0.7 \mathrm{a}\end{array}$ & $\begin{array}{r}28.3 \mathrm{a} \\
3.3 \mathrm{a} \\
0.1 \mathrm{~b} \\
16.8 \mathrm{a} \\
22.2 \mathrm{a} \\
2.8 \mathrm{~b} \\
2.7 \mathrm{ab} \\
53.6 \mathrm{a} \\
25.3 \mathrm{a} \\
17.9 \mathrm{a} \\
11.8 \mathrm{a} \\
3.7 \mathrm{a} \\
11.0 \mathrm{a} \\
3.6 \mathrm{a} \\
6.9 \mathrm{a} \\
5.6 \mathrm{a} \\
3.3 \mathrm{a} \\
5.1 \mathrm{a} \\
31.6 \mathrm{a} \\
8.9 \mathrm{a} \\
6.2 \mathrm{a} \\
5.9 \mathrm{a} \\
18.4 \mathrm{a} \\
11.1 \mathrm{a}\end{array}$ & $\begin{array}{l}0.5 \mathrm{a} \\
0.1 \mathrm{a} \\
1.0 \mathrm{a} \\
0.6 \mathrm{a} \\
1.0 \mathrm{~b} \\
0.4 \mathrm{a} \\
1.2 \mathrm{a} \\
1.6 \mathrm{~b} \\
1.9 \mathrm{a} \\
1.1 \mathrm{a} \\
0.1 \mathrm{~b} \\
0.4 \mathrm{a} \\
0.6 \mathrm{a} \\
0.4 \mathrm{a} \\
0.4 \mathrm{a} \\
0.2 \mathrm{a} \\
0.0 \mathrm{a} \\
0.0 \mathrm{a} \\
0.8 \mathrm{~b} \\
2.5 \mathrm{a} \\
0.1 \mathrm{a} \\
1.2 \mathrm{a} \\
2.3 \mathrm{a} \\
0.1 \mathrm{~b}\end{array}$ & $\begin{array}{r}12.1 \mathrm{a} \\
1.5 \mathrm{a} \\
9.2 \mathrm{a} \\
3.9 \mathrm{a} \\
7.6 \mathrm{~b} \\
8.7 \mathrm{a} \\
7.6 \mathrm{a} \\
24.2 \mathrm{~b} \\
13.0 \mathrm{a} \\
11.3 \mathrm{a} \\
2.3 \mathrm{~b} \\
1.9 \mathrm{a} \\
12.0 \mathrm{a} \\
3.6 \mathrm{a} \\
5.4 \mathrm{a} \\
4.8 \mathrm{a} \\
1.2 \mathrm{a} \\
0.6 \mathrm{a} \\
10.2 \mathrm{~b} \\
8.7 \mathrm{a} \\
0.9 \mathrm{a} \\
11.7 \mathrm{a} \\
20.1 \mathrm{a} \\
2.1 \mathrm{~b}\end{array}$ & $\begin{array}{l}0.7 \mathrm{a} \\
0.0 \mathrm{~b} \\
0.4 \mathrm{a} \\
1.5 \mathrm{a} \\
0.8 \mathrm{~b} \\
0.1 \mathrm{~b} \\
0.6 \mathrm{a} \\
1.5 \mathrm{c} \\
2.6 \mathrm{a} \\
0.6 \mathrm{c} \\
0.2 \mathrm{~b} \\
0.4 \mathrm{a} \\
0.6 \mathrm{~b} \\
0.1 \mathrm{a} \\
0.4 \mathrm{a} \\
0.1 \mathrm{~b} \\
0.1 \mathrm{~b} \\
0.3 \mathrm{ab} \\
1.1 \mathrm{~b} \\
1.1 \mathrm{c} \\
0.2 \mathrm{a} \\
0.4 \mathrm{a} \\
2.1 \mathrm{a} \\
0.2 \mathrm{a}\end{array}$ & $\begin{array}{r}18.8 \mathrm{~b} \\
0.7 \mathrm{~b} \\
3.5 \mathrm{a} \\
9.9 \mathrm{a} \\
8.8 \mathrm{c} \\
1.0 \mathrm{~b} \\
3.0 \mathrm{a} \\
35.0 \mathrm{c} \\
20.5 \mathrm{a} \\
8.2 \mathrm{~b} \\
3.4 \mathrm{~b} \\
1.8 \mathrm{a} \\
11.8 \mathrm{~b} \\
3.2 \mathrm{a} \\
4.4 \mathrm{a} \\
1.6 \mathrm{~b} \\
3.4 \mathrm{ab} \\
4.5 \mathrm{a} \\
18.0 \mathrm{~b} \\
5.1 \mathrm{a} \\
1.7 \mathrm{~b} \\
4.5 \mathrm{a} \\
24.3 \mathrm{a} \\
3.0 \mathrm{~b}\end{array}$ & $\begin{array}{l}1.4 \mathrm{~b} \\
0.2 \mathrm{a} \\
0.4 \mathrm{a} \\
1.8 \mathrm{a} \\
1.7 \mathrm{a} \\
0.1 \mathrm{~b} \\
0.6 \mathrm{a} \\
4.1 \mathrm{a} \\
3.3 \mathrm{a} \\
1.9 \mathrm{a} \\
0.5 \mathrm{a} \\
0.9 \mathrm{a} \\
0.8 \mathrm{a} \\
0.4 \mathrm{a} \\
0.5 \mathrm{a} \\
0.2 \mathrm{a} \\
0.5 \mathrm{a} \\
0.4 \mathrm{a} \\
2.2 \mathrm{a} \\
2.0 \mathrm{~b} \\
0.5 \mathrm{a} \\
1.1 \mathrm{a} \\
2.8 \mathrm{a} \\
0.5 \mathrm{a}\end{array}$ & $\begin{array}{r}24.8 \mathrm{a} \\
4.8 \mathrm{a} \\
3.5 \mathrm{a} \\
10.8 \mathrm{a} \\
13.7 \mathrm{a} \\
2.2 \mathrm{~b} \\
3.8 \mathrm{a} \\
41.3 \mathrm{~b} \\
20.4 \mathrm{a} \\
17.3 \mathrm{a} \\
6.0 \mathrm{~b} \\
3.8 \mathrm{a} \\
15.6 \mathrm{a} \\
4.7 \mathrm{a} \\
5.6 \mathrm{a} \\
5.6 \mathrm{a} \\
6.1 \mathrm{a} \\
3.7 \mathrm{a} \\
23.1 \mathrm{a} \\
8.3 \mathrm{a} \\
2.0 \mathrm{~b} \\
7.3 \mathrm{a} \\
29.3 \mathrm{a} \\
6.8 \mathrm{a}\end{array}$ & $\begin{array}{l}0.7 \mathrm{a} \\
0.1 \mathrm{~b} \\
0.2 \mathrm{a} \\
0.9 \mathrm{a} \\
1.0 \mathrm{~b} \\
0.3 \mathrm{a} \\
0.4 \mathrm{a} \\
2.3 \mathrm{~b} \\
2.2 \mathrm{a} \\
1.3 \mathrm{~b} \\
0.5 \mathrm{a} \\
0.3 \mathrm{a} \\
0.2 \mathrm{c} \\
0.3 \mathrm{a} \\
0.2 \mathrm{~b} \\
0.2 \mathrm{a} \\
0.1 \mathrm{~b} \\
0.2 \mathrm{~b} \\
1.1 \mathrm{~b} \\
3.0 \mathrm{a} \\
1.0 \mathrm{a} \\
0.9 \mathrm{a} \\
0.4 \mathrm{~b} \\
0.1 \mathrm{a}\end{array}$ & $\begin{array}{c}25.0 \mathrm{a} \\
1.2 \mathrm{~b} \\
3.0 \mathrm{a} \\
11.0 \mathrm{a} \\
11 . \mathrm{b} \\
9.3 \mathrm{a} \\
3.6 \mathrm{a} \\
49.1 \mathrm{a} \\
21.9 \mathrm{a} \\
16.4 \mathrm{a} \\
8.9 \mathrm{a} \\
2.7 \mathrm{a} \\
9.4 \mathrm{~b} \\
3.9 \mathrm{a} \\
3.7 \mathrm{a} \\
6.1 \mathrm{a} \\
1.1 \mathrm{~b} \\
3.0 \mathrm{a} \\
20.8 \mathrm{ab} \\
10.6 \mathrm{a} \\
6.5 \mathrm{a} \\
10.4 \mathrm{a} \\
10.3 \mathrm{~b} \\
4.5 \mathrm{~b}\end{array}$ \\
\hline $\begin{array}{l}\text { Annuals } \\
\text { Bromus japonicus } \\
\text { Collinsia parviflora } \\
\text { Collomia linearis } \\
\text { Epilobium paniculatum } \\
\text { Foeniculum vulgare } \\
\text { Lactuca serriola }\end{array}$ & $\begin{array}{l}0.1 \mathrm{a} \\
0.1 \mathrm{~b} \\
0.2 \mathrm{~b} \\
0.1 \mathrm{~b} \\
0.2 \mathrm{a} \\
0.0 \mathrm{~b}\end{array}$ & $\begin{array}{l}3.6 \mathrm{a} \\
3.0 \mathrm{~b} \\
6.8 \mathrm{~b} \\
2.4 \mathrm{~b} \\
5.0 \mathrm{a} \\
1.2 \mathrm{~b}\end{array}$ & $\begin{array}{l}0.1 \mathrm{a} \\
0.1 \mathrm{~b} \\
0.4 \mathrm{~b} \\
0.2 \mathrm{~b} \\
0.3 \mathrm{a} \\
0.1 \mathrm{~b}\end{array}$ & $\begin{array}{r}1.6 \mathrm{a} \\
2.3 \mathrm{~b} \\
11.3 \mathrm{~b} \\
5.9 \mathrm{~b} \\
7.8 \mathrm{a} \\
2.7 \mathrm{~b}\end{array}$ & $\begin{array}{l}0.1 \mathrm{a} \\
0.4 \mathrm{a} \\
0.9 \mathrm{a} \\
1.3 \mathrm{a} \\
0.4 \mathrm{a} \\
0.8 \mathrm{a}\end{array}$ & $\begin{array}{r}2.6 \mathrm{a} \\
11.5 \mathrm{a} \\
22.9 \mathrm{a} \\
27.3 \mathrm{a} \\
8.7 \mathrm{a} \\
17.5 \mathrm{a}\end{array}$ & $\begin{array}{l}0.0 \mathrm{~b} \\
0.0 \mathrm{c} \\
0.3 \mathrm{~b} \\
0.0 \mathrm{~b} \\
0.1 \mathrm{~b} \\
0.1 \mathrm{~b}\end{array}$ & $\begin{array}{l}0.3 \mathrm{~b} \\
0.3 \mathrm{~b} \\
6.2 \mathrm{~b} \\
0.3 \mathrm{c} \\
2.4 \mathrm{~b} \\
1.1 \mathrm{~b}\end{array}$ & $\begin{array}{l}0.0 \mathrm{~b} \\
0.3 \mathrm{a} \\
0.6 \mathrm{a} \\
0.7 \mathrm{a} \\
0.5 \mathrm{a} \\
0.7 \mathrm{a}\end{array}$ & $\begin{array}{r}1.0 \mathrm{~b} \\
9.4 \mathrm{a} \\
14.8 \mathrm{a} \\
9.8 \mathrm{~b} \\
14.8 \mathrm{a} \\
11.9 \mathrm{a}\end{array}$ & $\begin{array}{l}0.2 \mathrm{a} \\
0.2 \mathrm{~b} \\
0.5 \mathrm{a} \\
0.9 \mathrm{a} \\
0.1 \mathrm{~b} \\
0.2 \mathrm{~b}\end{array}$ & $\begin{array}{r}6.5 \mathrm{a} \\
7.0 \mathrm{a} \\
20.0 \mathrm{a} \\
15.5 \mathrm{a} \\
2.3 \mathrm{~b} \\
8.3 \mathrm{a}\end{array}$ \\
\hline
\end{tabular}

Number to the left is percent coverage; the number to the right is percent frequency.

2Means within a row followed by same letters are not significantly different $(P<0.1)$. 
were relatively uniform (C.V. $=38.1$; however, samples were highly variable $(0$ to $14 \mathrm{~cm})$. Site inspection the day after treatment revealed that the organic material on high intensity sites continued to smolder. On the average, duff combustion on low intensity sites was not as complete as on the high intensity sites. However, duff reduction is not solely dependent on fire intensity. Other factors such as duff moisture also influence duff reduction. Therefore, a burn with a high fire intensity does not necessarily decrease duff more than a burn with a low fire intensity (Bakken 1981). Graminoid response appeared to vary with the amount of duff burned, regardless of the fire line intensity.

Fire line intensity was calculated as the product of rate of spread, fuel weight consumed per unit area, and heat of combustion (Van Wagner 1973). Flame length was calculated (Rothermel and Deeming 1980). The latter was rejected because of the high variability in flame length.

It was thought that a high intensity fire would also relate to a high amount of duff removed; however, this did not usually occur. Flame length is determined by the amount of fire fuel consumed by the advancing flame front. Large fuels and duff do not burn until after the flame front passes. Both the large fuel and duff burn according to their moisture contents. Fire intensity is determined by the fine fuel quantity and moisture content. Fine fuel moisture is independent of the large fuel and duff moistures and, therefore, fire intensity may not be related to the duff consumed. Only when both the fine fuel and duff moistures were low did the fire intensity relate to the duff consumed. However, we observed plots where the intensity was low and the duff consumption was high and also where the intensity was high and little duff was removed.

For example, the fire on one low intensity site had an average flame length of $1.2 \mathrm{~m}$, a rate of spread of $0.9 \mathrm{~m} / \mathrm{min}$, little downed woody fuel consumed, an intensity of $148 \mathrm{kcal} / \mathrm{m}-\mathrm{s}$, yet an average $4.5 \mathrm{~cm}$ of duff was removed. On a high intensity site, the flame length was $0.5 \mathrm{~m}$, the backing rate of spread was $0.3 \mathrm{~m} / \mathrm{min}$, woody fuel consumed was $2.1 \mathrm{~kg} / \mathrm{m}^{2}$, the fire line intensity was 130 $\mathrm{kcal} / \mathrm{m}-\mathrm{s}$, and the amount of duff removed was $4.8 \mathrm{~cm}$. In this example, fire line intensity and duff consumed were similar; however, flame length and rate of spread were vastly different. The response of the graminoids was similar in the 2 fires even though the fire behavior was different.

Canopy coverage for shrubs was unaffected by treatment. There was no change in cover among treatments for snowberry (Symphoricarpos albus) (Table 1), the dominant shrub on the sampled plots. This may have masked the response of other, less abundant shrubs. Fires that consumed only a small portion of the duff layer did not affect shrub cover.

The forb composition changed little due to the different fire treatments. Jaccard's Index of Similarity, for the unburned sites versus sites treated with high intensity fires, ranged from 73 to 82 , implying that the flora on differently treated sites were similar. The sites also had a large number of total forb species present. Increases in low seral species such as fireweed and collomia were offset by decreases in higher seral species such as strawberry and slender cinquefoil. Consequently, total forb cover remained fairly uniform between intensities and few significant changes occurred as a result of the fires (Table 2).

An 11 to $35 \%$ reduction in overstory basal area by selective logging stimulated a continual increase in shrub canopy coverage over the 3-year period on all burned and unburned sites. An increase in the amount of light striking the forest floor, together with decreased competition for moisture between shrubs and trees, enabled the shrub layer to expand. Armour (1982) noted a similar phenomenon in lodgepole pine (Pinus contorta) stands killed by mountain pine beetle (Dendroctonus pseudotsugae). Apparently, competition for moisture and light was reduced with the removal of the overstory.

The pattern exhibited by grasses and forbs was very different from the observed recovery pattern for shrubs. One would expect a continual increase in canopy coverage for both grass and forbs over a 3-year period. In broadcast-burned clearcuts, Zamora (1982) reported the greatest increase in total canopy coverage occurred between the second and third year after burning. The volcanic ash from Mt. St. Helens may have been responsible for the abnormal response observed in this study. The yield for 3 cultivated gains near Farmington, Wash., (approximately 28 $\mathrm{km}$ southwest of the study area) was similar to the response exhibited by the herb layer. Evidently, the ash deposit sealed the soil surface and effectively reduced the evaporative component of the water budget. Thus, the abrupt increase in cover may have been the result of an increase in available moisture in the upper soil profile. This cap effect was broken up by the following year and was not apparent in 1981 .

\section{Conclusions}

Two conclusions may be drawn from the results of this study. First, the most important factor affecting vegetational change following understory burning in ponderosa pine in this habitat type is the preburn plant community. The abundance of many species remained relatively uniform under the variation of fire intensities studied. Graminoids tended to increase in canopy coverage but not in frequency. This probably indicates that some mortality occurred but the increased growth of the plants on the burned area compensated for this loss. Total forb cover remained fairly constant across treatments but the relative amounts of species changed. The composition present at the time of the fire is consequently an important factor in the successional change after the fire.

Second, the change in graminoid abundance appeared to be related more directly to the amount of duff consumed than to the fire line intensity and flame length. Thus, when predicting vegetation changes in response to understory burning, one needs to be aware that the response of the understory herbaceous and woody plants in ponderosa pine communities is independent of flame length and fire line intensity and is dependent on the amount of woody fuel and duff consumed. The majority of understory plants have the tops killed by the flames passing over them. Mortality is apparently induced when sufficient heat is diverted to their regenerating organs which are near or below the ground surface. The heat reaching the regenerating organs and roots is generated after the flame front has passed and results from the longer duration of heat from heavier fuels. the overstory ponderosa pine trees are, of course, affected by the intensity and flame lengths since their regenerating organs are aboveground.

\section{Literature Cited}

Armour, C.D. 1982. Fuel and vegetation succession in response to mountain pine beetle epidemics. M.S. Thesis, Univ. of Idaho, Moscow.

Bakken, S. 1981. Predictions of fire behavior, fuel reduction, and tree damage from understory prescribed burning in the Douglas-fir/ninebark habitat type of northern Idaho. M.S. Thesis, Univ, of Idaho, Moscow.

Biswell, H.H., H.R. Kallander, R. Komarack, R.J. Vogl, and H. Weaver. 1973. Ponderosa fire management. Tall Timbers Res. Sta. Misc. Pub. 2. Tallahassee, Fla.

Canfield, H.R. 1941. Application of the line intercept method in sampling range vegetation. J. Forest. 39:388-394.

Cattelino, P.J., I.R. Nobel, R.O. Slatyer, and S.R. Kessell. 1979. Predicting the multiple pathways of plant succession. Environ. Manage. 3:41-50.

Daubenmire, R. 1959. A canopy-coverage method of vegetation analysis. Northwest Sci. 33:43-66. 
Daubenmire, R., and J.B. Daubenmire. 1968. Forest vegetation of eastern Washington and northern Idaho. Washington State Agr. Sta. Tech. Bull. No. 60.Pullman.

Flint, H.R. 1925. Fire resistance of northern Rocky Mountain conifers. Idaho Forest 7:7-10.

Helwig, J.T., and K.A. Council, eds. 1979. SAS users guide 1979 edition. SAS Institute Inc., Raleigh, N.C.

Hitcheock, C.L., and A. Cronquist. 1973. Flora of the Pacific Northwest. Univ. of Washington Press, Seattle.

Hooker, L.L. 1972. Effects of prescribed burning on soils and vegetation of seral brush communities in the Lochsa River region of northern Idaho. M.S. Thesis, Univ. of Idaho, Moscow.

Keown, L.D. 1978. Fire management in the Selway-Bitterroot Wilderness, Moose Creek Ranger District, Nezperce National Forest. USDA Forest Serv. Nezperce National Forest.

Larsen, J.A. 1929. Fires and forest succession in the Bitterroot Mountains of northern Idaho. Ecology 10:67-76.

Leege, T.A. 1968. Prescribed burning for elk in northern Idaho. Tall Timbers Fire Ecol. Conf. Proc. 8:235-253.

Lyon, L.J. 1971. Vegetal development following prescribed burning of Douglas-fir in south central Idaho. USDA Forest Serv. Res. Pap. INT-105.

Lyon, L.J., and P.F. Stickney. 1976. Early vegetal succession following large Rocky Mountain wildfires. Tall Timbers Fire Ecol. Conf. Proc. 14:355-373.
Mueller-Domboil, D., and H. Ellenberg. 1974. Aims and methods of vegetation ecology. J. Wiley \& Sons, New York.

Rothermel, R.C., and J.E. Deeming. 1980. Measuring and interpreting fire behavior for correlation with fire effects. USDA Forest Serv. Gen. Tech. Rep. INT-93.

Schubert, G.H. 1974. Silviculture of southwestern ponderosa pine: The state of our knowledge. USDA Forest Serv. Res. Pap. RM-23.

Schultz, A.M., and H.H. Blawell. 1959. Effect of prescribed burning and other seedbed treatments on ponderosa pine seedling emergence. J. Forest. 57:816-817.

Van Wagner, C.E. 1973. Height of crown scorch in forest fires. Can. J. Forest. Res. 3:373-378.

Wright, H.A. 1978. The effects of fire on vegetation in ponderosa pine forests. A state-of-the-art review. Texas Tech Univ., Coll. Agr. Sci. Pub. T-9-199, Lubbock.

Wright, H.A., and A.W. Balley. 1982. Fire ecology: United States and southern Canada. J. Wiley \& Sons, New York.

Zamora, B.A. 1982. Understory development in forest succession: An example from the Inland Northwest. p. 63-69. In: Forest Succession and Stand Development Research in the Northwest Proc., ed. J.E. Means. For. Res. Lab., Oregon State Univ., Corvallis.

\section{Page Charges}

Members of the Society for Range Management will receive a 35\% discount on page charges for the first three published pages of articles in the Journal of Range Management. In the case of articles with multiple authors, membership by one author is sufficient to obtain the discount. Beginning in June, 1984, page charges will be $\$ 100$ per page; the membership discount will make charges $\$ 65$ per page for each of the first three published pages. Publication is not contingent on membership in the Society nor have other policies changed regarding the assessing of page charges and reprint costs.

\section{Style Manual for $\boldsymbol{J R M}$}

A Handbook and Style Manual for the Journal of Range Management has been prepared to assist authors in the preparation of manuscripts for the Journal. Although not intended as an exhaustive presentation on manuscript presentation, it was prepared with the less-experienced author in mind. Manuscripts submitted after 30 June, 1984 , and not conforming to $J R M$ style as designated in the manual will be returned to authors for correction before being entered into the review process. The manual will remain in effect for at least 2 years, at which point it will be reviewed and revised as necessary.

Copies of the Handbook and Style Manual (12 pages) are available from the Society for Range Management, 2760 West Fifth Avenue, Denver, CO 80204, at $\$ 1.25$ postpaid for single copies; $\$ 1.00$ each for 2 or more.

Pages from the manual will be published on the inside back cover of the Journal of Range Management. 